\title{
Deceptive Imprinting
}

National Cancer Institute

\section{Source}

National Cancer Institute. Deceptive Imprinting. NCI Thesaurus. Code C19131.

Refers to a mechanism leading to a fixed state of immunity that fails to adapt to a chang ing pathogen and has lost the ability to discriminate antigen after the establishment of a strong primary immune response. 